\title{
Perceived health status and adherence to haemodialysis by End Stage Renal Disease patients: A case of a Central hospital in Zimbabwe.
}

\author{
Geldine Chironda 1 , Ancia Manwere ${ }^{2}$, Rudo. Nyamakura ${ }^{3}$, Tirivanhu Chipfuwa ${ }^{4}$, \\ Busisiwe Bhengu ${ }^{5}$ \\ ' (School of Nursing and Public Health, University of Kwazulu Natal, South Africa), 2(Department of Health \\ sciences, Bindura University of Science Education, Zimbabwe), ${ }^{3}$ (Department of Nursing Science, University of \\ Zimbabwe, Zimbabwe), ${ }^{4}$ (Department of Health sciences, Bindura University of Science Education, \\ Zimbabwe), 5 (School of Nursing and Public Health, University of Kwazulu Natal, South Africa)
}

\begin{abstract}
Poor adherence to haemodialysis among End Stage Renal Disease (ESRD) patients has been a major problem globally. Perceived physical health appears to be the major contributing factor to poor adherence to haemodialysis. Improving the adherence to haemodialysis among ESRD patients improves their quality of life. A descriptive correlational study was conducted to examine the relationship between perceived physical health and level of adherence to haemodialysis among ESRD patients. The study was conducted at a central hospital in Harare, Zimbabwe. A total of 85 ESRD patients who met the inclusion criteria were selected using simple random sampling. Data was collected using an interview schedule with three sections namely: the demographic data, adherence to haemodialysis and perceived physical health. Descriptive statistics, Pearson's correlation coefficient and linear regression were used to analyse the data. Eighty - three (97.6\%) demonstrated low adherence to haemodialysis. Eighty - two (96.5\%) perceived their physical health to be average. The Pearson correlation coefficient ( $r=-.44 p<.01)$ showed a weak negative significant relationship. Perceived physical health had an impact of $19.7 \%$ on adherence to haemodialysis $\left(R^{2}\right.$ 0.197). The findings predict that as perceived physical health increase, adherence to haemodialysis decrease among ESRD patients. Medical surgical nurses should intensify individualized client programmes to correct ESRD clients' perceptions and identify more contributory factors to poor adherence to haemodialysis.
\end{abstract}

Keywords: adherence, end stage renal disease, haemodialysis, perceived physical health.

\section{Introduction}

End stage renal disease (ESRD) is the complete or almost complete failure of the kidneys to function at a level that is necessary for day to day life. In ESRD, renal failure has progressed to the point where kidney function is less than ten percent of normal (Tolkoff-Rubin, 2008). Louis, Davies, \& White (2001) defined ESRD as a life threatening condition and survival is only with haemodialysis, a form of renal replacement therapy. According to Tolkoff-Rubin (2008) without haemodialysis death will occur from the build up of fluids and waste products in the body. End stage renal disease is increasing worldwide at an annual growth rate of $8 \%$ and millions of people around the world suffer from this disease (Ayobiesu \& Ayodele, 2005).

The prevalence of kidney disease ( ESRD) is estimated to be 8-16\% worldwide and similar estimates of the burden of Kidney disease has been reported around all continents (Vivekanand, etal, 2013). In Africa, there is consistent increase in the Incidence of newly diagnosed individuals with ESRD and several studies report a prevalence of ESRD of about 10\% (Naicker, 2009, Singh et al, 2013 \& Barsoum, 2013). Poor adherence with heamodialysis is seen in almost half of the patients treated for ESRD yet heamodialysis is a life saving procedure for these patients (Richard, 2006). Avu (2009) defined heamodialysis as a procedure that is perfomed routinely on end stage renal disease patients and involves removing waste substances and fluid that are normally eliminated by the kidneys from the blood. As with most chronic illnesses, poor adherence to prescribed heamodialysis regimen is a pervasive problem in the ESRD population (Mitch, 2007). According to Ayobiesu and Ayodele (2005), poor adherencce is seen in end stage renal disease patients' undergoing haemodialysis yet haemodialysis is a life saving procedure for these patients. Therefore, adherence to heamodialysis is most important in ESRD patients undergoing heamodialysis.

\subsection{Problem statement}

In Zimbabwe, there appears to be reduced adherence to heamodialysis among ESRD patients. Statistics at one local haemodialysis unit in Harare, Zimbabwe show that in 2007 and 2008, 55\% of ESRD patients adhered to heamodialysis during the initial month and the adherence levels deteriorated with time. About $20 \%$ adhered to haemodialysis for 2 consecutive months, $8 \%$ for 3 consecutive months and none of the patients adhere to 
heamodialysis for more than 12 consecutive months (Parirenyatwa Hospital Records, 2007-2008). At a second haemodialysis centre in Harare, almost similar patterns of low adherence to haemodialysis by ESRD patients were noted for the 2007 to 2008 period. Although 75\% of ESRD patients adhered to heamodialysis for 3 consecutive months, 50\% adhered for 3 to 6 months and only 37\% adhered for more than 6 months (Harare Haemodialysis Centre Records, 2007 - 2008 unpublished).

The deteriorating adherence rates to this important therapy which calls largely for patients' motivation are a cause for concern. Patient's apparent willingness to follow medication regimens or to adhere to medical treatment regimens reflects a significant barrier to the effective management of most chronic disorders (Mitch, 2007). However, there are no reliable statistics that describe adherence to dialysis of ESRD patients in all African countries. However, given the poor socioeconomic status, reduced adherence to haemodialysis seems to be higher in all African countries. In Nigeria, 70.8\% of the patients are able to adhere to dialysis for one month, $12.7 \%$ between 3 and 6 months and $5.1 \%$ between 7 and 12 moths. Only $1.9 \%$ adheres to haemodialysis for more than 12 months (Barsoum, 2003).

Reduced adherence to heamodialysis has been reported to be the most common cause of failure to respond to medications and heamodialysis itself (Alebiosu \& Ayodele, 2005). Tolkoff-Rubin (2008) further emphasized that poor adherence to haemodialysis increases the risk of complications in ESRD patients. Complications include chronic anaemia, brain dysfunction, congestive heart failure, decreased function of white blood cells, excessive bleeding, infections, weakness of bones and pulmonary complications. Non adherence also leads to additional and often unnecessary tests, dosage, adjustments, and changes in treatment plan, emergency department visits and hospitalization which ultimately results in increased cost of medical care (Mitch, 2007). Several studies have shown that poor adherence with heamodialysis treatment is associated with increased mortality and morbidity. The mortality risk increases when the serum phosphorus level chronically exceeds $6.5 \mathrm{mg} / \mathrm{dl}$ (Ayobiesu \& Ayodele, 2005). Without adequate dialysis, death occurs from build up of fluids and waste products in the body (Patel, 2008). According to the 2010 Global Burden of Disease Study, ESRD was ranked $27^{\text {th }}$ in the list of causes of total number of Global deaths in 1990 with age standardized annual death rate of 15.7 per 100000 , but rose to $18^{\text {th }}$ in 2010 with annual death rate of 16.3 per 100000 (Lozano, Naghavi \& Foreman, 2013)

In Zimbabwe, a mortality rate of 58\% was recorded in the year 2007 to 2008 (Parirenyatwa Renal Unit records, 2008 unpublished). Therefore adherence to haemodialysis of end stage renal disease patients is an area of concern locally, regionally and globally in medical surgical nursing. The most likely contributing factors to reduced adherence to haemodialysis appear to be perceived physical health status. Informal observations indicate that tendencies to omit haemodialysis sessions due to a feeling of well being among ESRD patients at a central hospital in Zimbabwe appear to be on the increase. Physical health is the overall condition of a living organism at a given time, the condition of optimal wellbeing and the ability of the body to carry out all the activities of living (Kagaku, 20006).

It appears end stage renal disease patients have a false sense of well being and deliberately choose not to report for the next dialysis session until symptoms worsen. The way end stage renal disease patients perceive and react to their physical health appears to affect their adherence to heamodialysis. Elsevier (2009) stated that patient's beliefs about their physical health are important because they influence adherence and adjustment to heamodialysis. Koudi (2004) expressed similar sentiments and stated that ESRD patients display physical health disturbances as well as non adherence to heamodialysis treatment.

According to Louise, Davies, \& White (2001), complications of end stage renal disease have been found to have a significant impact on the physical health of these patients. It is well documented that the health status of end stage renal disease patients is worse than that of the general population. For this reason, assessment of physical wellbeing of end stage renal disease patients has received considerable attention. In this study, it has been found important to recognize how well ESRD patients are able to function and how they feel about their day to day life. Thus, understanding the impact of end stage renal disease and associated heamodialysis treatment on functioning and wellbeing in physical dimensions of life is essential. Research and documented evidence on perceived physical health in end stage renal disease patients is critical to medical surgical nurse practitioners but is lacking in Zimbabwe. Therefore, the investigator sought to characterize perceived physical health in ESRD population.

Previous studies have implicated perceived physical health as an important correlate of adherence behaviour in other chronic illnesses groups but little research has examined this relationship in a heamodialysis population with ESRD. Therefore, the investigator sought to fill this gap by examining the relationship between perceived physical health and adherence to heamodialysis among end stage renal disease patients. Medical surgical nurses would therefore disseminate appropriate and relevant information through focused health education and individual client discussions to foster adherence to haemodialysis thus improving effective management of the population. 


\subsection{Purpose of the study}

The purpose of this study was to examine the relationship between level of adherence to heamodialysis and perceived health status of ESRD patients at a central hospital in Harare. The study determined and described the level of adherence to haemodialysis and perceived physical health of ESRD patients prior to examining the relationship between the two variables.

1.4 Objectives

- To determine level of adherence to heamodialysis among ESRD patients at a haemodialysis centre in Harare.

- To determine level of perceived physical health of ESRD patients on heamodialysis at a haemodialysis centre in Harare.

- To examine the relationship between perceived physical health and adherence to heamodialysis among ESRD patients at a haemodialysis centre in Harare.

\subsection{Conceptual framework}

The conceptual framework selected for this study was based on Roper, Logan and Tierney's Activities of Living Model.

\section{Materials And Methods}

The research design used in this study was the descriptive correlational design. The study was conducted at Parirenyatwa Haemodialysis Centre in Zimbabwe. The haemodialysis centre is situated in a tertiary referral centre. It was also the only public haemodialysis centre functioning in the country at the time of the study. Focusing at this haemodialysis centre hence posed a likelihood of capturing ESRD patients from all over the country. A sample of 85 ESRD adult patients was selected using simple random sampling with replacement on the basis of inclusion and exclusion criteria. The tool used for this study was the structured interview schedule consisting of section 1 (Socio-demographic variables such as Age, Gender, Marital status, family income, Duration of Renal failure illness), Section 11(consisting of 4 items related to adherence to haemodialysis) and Section 111(consisting of 16 items related to Perceived Physical health). Face-to-face interview was used to collect data. The content validity of the structured interview schedule was ensured by submitting the tool to the experts in the field of renal failure and haemodialysis. A pilot study was conducted on adult ESRD patients who met the inclusion criteria at a Private haemodialysis Centre (Harare Haemodialysis Centre). Cronbach's alpha was the psychometric test applied to measure the internal consistency of the instrument. The reliability of the tool was calculated and it was 0.79 .

\section{Results And Findings}

A total of eighty-five ( $\mathrm{n}=85$ ) ESRD patients from Parirenyatwa Haemodialysis Centre participated in the study.

\subsection{Section 1: Demographic Characteristics}

The age range for the 85 respondents was 19 to 60 years. The mean age was 43.67 years, median age was 44 years and modal age was 40 years. The age categories are shown in Table 1 . The majority of the respondents $52(61.2 \%)$ were male. Thirty six $(42.4 \%)$ of the respondents had secondary level education, $11(12.9 \% 0$ had advanced level education and 33(38.8\%) had tertiary level education. The majority of the respondents 50(58.8\%) had no income. Most of the respondents 66(77.6\%) had been on haemodialysis for more than a year (Table 1).

Table 1: Demographic Characteristics $(n=85)$

\begin{tabular}{lcc}
\hline Variable & Frequency (n) & Percentage (\%) \\
\hline Age categories (years) & 7 & 7.1 \\
$19-30$ & 28 & 32.9 \\
$31-40$ & 32 & 37.6 \\
$41-50$ & 18 & 21.2 \\
$51-60$ & & \\
Level of Education & 5 & 5.9 \\
Primary & 36 & 42.4 \\
Secondary level & 11 & 12.9 \\
Advanced level & 33 & 38.8 \\
Tertiary level & & \\
Gender & 52 & 61.2 \\
Male & &
\end{tabular}


Female

Duration of haemodialysis

3 months to 1 year

1.5 to 2 years

2.5 to 3 years

3.5 t0 4 years

4.5 to 5 years

More than 5 years
33

19

19

18

1

18
38.8

22.4

22.4

21.2

1.2

11.8

21.2

\section{Perceived Physical Health}

Table 2 shows that $32(37.6 \%)$ of the respondents perceived their health status as poor, 41(48.2\%) perceived it as fair and 1(1.2\%) perceived it as very good and excellent respectively. Furthermore, $40(47.1 \%)$ of the respondents perceived their physical health as worse, 34(40\%) perceived it has not changed and 11(13\%) perceived it as better in the past 3 months.

Table 2: Perceived Physical Health $(n=85)$

\begin{tabular}{llc}
\hline Variable & Frequency $(\mathbf{n})$ & Percentage $(\%)$ \\
\hline Health Status & 32 & 37.6 \\
Poor & 41 & 48.2 \\
Fair & 10 & 11.8 \\
Good & 1 & 1.2 \\
Very good & 1 & 1.2 \\
Excellent & & \\
Physical health compared to 3 months ago & 40 & 47.1 \\
Worse & 34 & 40.0 \\
No change & 11 & 13.0 \\
Better & & \\
\hline
\end{tabular}

Perceived Physical Health (Activities of Daily Living)

Table 3 shows that most of the respondents 71(83.5\%) perceived themselves as having problems with elimination, mobilisation 51(60.0\%), sleeping 51(60.0\%), resting and working 51(60.0\%) and eating and drinking 51(60.0\%).

Table 3: Perceived Physical Health (Activities of Daily Living) $(\mathbf{n}=\mathbf{8 5})$

\begin{tabular}{|c|c|c|}
\hline Variable & Frequency (n) & Percentage $(\%)$ \\
\hline \multicolumn{3}{|c|}{ Problems with Elimination (urination) } \\
\hline Most of the time & 71 & 83.5 \\
\hline Some of the time & 9 & 10.6 \\
\hline A little of the time & 1 & 1.2 \\
\hline I never do & 4 & 4.7 \\
\hline \multicolumn{3}{|c|}{ Problems with Mobilization } \\
\hline Most of the time & 51 & 60.0 \\
\hline Some of the time & 18 & 21.2 \\
\hline A little of the time & 8 & 9.4 \\
\hline I never do & 8 & 9.4 \\
\hline \multicolumn{3}{|c|}{ Problems with Sleeping and Resting } \\
\hline Most of the time & 51 & 60.0 \\
\hline Some of the time & 21 & 24.7 \\
\hline A little of the time & 7 & 8.2 \\
\hline I never do & 6 & 7.1 \\
\hline \multicolumn{3}{|c|}{ Problems with Working } \\
\hline Most of the time & 51 & 60.0 \\
\hline Some of the time & 21 & 24.7 \\
\hline A little of the time & 7 & 8.2 \\
\hline I never do & 6 & 7.1 \\
\hline \multicolumn{3}{|c|}{$\begin{array}{l}\text { Problems with Cleansing Personal } \\
\text { and Dressing }\end{array}$} \\
\hline Most of the time & 26 & 30.6 \\
\hline Some of the time & 38 & 44.7 \\
\hline
\end{tabular}


A little of the time

\section{8}

13

I never do

Problems with eating and drinking

Most of the time

Some of the time

A little of the time

I never do
9.4

15.3

69.4

18.8

3.5

8.2

\section{Perceived physical health}

Total perceived physical health scores ranged from 25 to 53 out of the possible score of 67 . The majority of the respondents $53(62.4 \%)$ perceived their physical health as poor by scoring below the average perceived physical health score of 33.5. Only 3(3.5\%) perceived their physical health to be good (Table 4).

Table 4: Total perceived physical health scores out of 67

\begin{tabular}{lcc}
\hline Score & Frequency $(\mathbf{n})$ & Percentage $(\%)$ \\
\hline 25 & 5 & 5.9 \\
26 & 5 & 5.9 \\
27 & 5 & 5.9 \\
28 & 6 & 7.1 \\
29 & 5 & 5.9 \\
30 & 7 & 8.2 \\
31 & 3 & 3.5 \\
32 & 6 & 7.1 \\
33 & 11 & 12.9 \\
34 & 6 & 7.1 \\
35 & 5 & 5.9 \\
36 & 3 & 3.5 \\
38 & 3 & 3.5 \\
39 & 3 & 3.5 \\
41 & 2 & 2.4 \\
42 & 4 & 4.7 \\
44 & 1 & 1.2 \\
45 & 1 & 1.2 \\
46 & 1 & 1.2 \\
48 & 1 & 1.2 \\
53 & 1 & 1.2
\end{tabular}

\section{Adherence to Haemodialysis}

Table 5 shows that $6(7.1 \%)$ of the respondents attended haemodialysis sessions all the time and the majority $52(38.9 \%)$ missed most of the haemodialysis sessions.

Table 5: Adherence to Haemodialysis $(\mathbf{n}=85)$

\begin{tabular}{|c|c|c|}
\hline Variable & Frequency (n) & Percentage $(\%)$ \\
\hline \multicolumn{3}{|c|}{ Extent of attending Haemodialysis } \\
\hline \multicolumn{3}{|l|}{ Sessions in the past 3 months } \\
\hline All the time & 6 & 7.1 \\
\hline Missed a few sessions & 27 & 31.8 \\
\hline Missed most of the sessions & 52 & 61 \\
\hline \multicolumn{3}{|c|}{ Rescheduled prescribed haemodialysis } \\
\hline \multicolumn{3}{|l|}{ Sessions in the past 3 months } \\
\hline None & 1 & 1.2 \\
\hline Once & 27 & 31.8 \\
\hline Twice & 48 & 56.5 \\
\hline More than twice & 9 & 10.6 \\
\hline \multicolumn{3}{|c|}{ Immediate arrangements for haemodialysis } \\
\hline \multicolumn{3}{|c|}{ Sessions after missing in the past 3 moths } \\
\hline Always & 55 & 64.7 \\
\hline Sometimes & 22 & 25.9 \\
\hline
\end{tabular}


Table 6 shows that the majority of the respondents 44(51.8\%) agreed that they did not adhere to haemodialysis because they were feeling physically fit and 30(35.3\%) reported that it was not necessary to follow haemodialysis schedules because of feeling generally well.

Table 6: Reasons for Non-adherence to Haemodialysis $(\mathbf{n}=85)$

\begin{tabular}{lcc}
\hline Reason & Frequency $(\mathbf{n})$ & Percentage $(\%)$ \\
\hline $\begin{array}{l}\text { Missed haemodialysis session } \\
\text { Because of feeling physically fit }\end{array}$ & \\
Not at all & 13 & 15.3 \\
Slightly agree & 18 & 21.2 \\
Agree & 44 & 51.8 \\
Strongly agree & 10 & 11.8 \\
Not necessary to follow haemodialysis & & \\
Schedule because of feeling generally well & 16 & 18.8 \\
Not at all & 23 & 27.1 \\
Slightly agree & 30 & 35.3 \\
Agree & 16 & 18.8 \\
Strongly agree & & \\
\hline
\end{tabular}

\section{Total Adherence score}

The highest total adherence score was 11 and the lowest score was 0 . A score of 0 meant nonadherence at all and a score of 11 meant excellent adherence. Table 7 shows that the highest attained total adherence score was 10 out of 11 and this was achieved by only 1(1.2\%) of the respondents. Eighty-three $(97.6 \%)$ of the respondents had a score of 8 and below out of the possible 11 scores demonstrating low levels of adherence to haemodialysis (Table 7).

Table 7: Total Adherence score out of 11

\begin{tabular}{lcc}
\hline Score & Frequency $(\mathbf{n})$ & Percentage $(\%)$ \\
\hline 1 & 1 & 1.2 \\
2 & 1 & 1.2 \\
4 & 7 & 8.2 \\
5 & 25 & 29.4 \\
6 & 28 & 32.9 \\
7 & 11 & 12.9 \\
8 & 10 & 11.8 \\
9 & 1 & 1.2 \\
10 & 1 & 1.2 \\
\hline
\end{tabular}

Relationship between Perceived Physical Health and Adherence to Haemodialysis

The results shows a weak negative correlation $(\mathrm{r}=-0.44, \mathrm{p}<.01)$. Therefore, as people perceive their physical health as getting better, adherence to haemodialysis slightly decreases. A linear regression result shows that $\mathrm{R}^{2}$ is 0.197 which means that the effect of perceived health status accounts for $19.7 \%$ of the variance in adherence to haemodialysis.

\section{Discussion}

This study was to examine the relationship between perceived physical health status and adherence to haemodialysis of ESRD patients. This will help nurses identify gaps in their health education on patients on haemodialysis. The ultimate goal is to improve patients' adherence to haemodialysis hence effective management of ESRD population, consequently their quality of life.

\subsection{Sample demographics}

The sample demographics related to gender showed that more males 52(61.2\%) presented with end stage renal disease than females 33(38.8\%). These findings are in contrast with Cucor, (2007), Cater, Coons, Mapes, Kallich \& Hays (2004), who noted that there are more females on heamodialysis with end stage renal disease than males. According to Cucor (2007), women composed 53\% of the sample while men composed $47 \%$ of their sample. 
The study showed that age ranged from nineteen years 19 to 60 years with the mean age of 43.67 , median age of 44 and modal age of 40 . These results reinforce the study by Hanly (2006) which showed that the mean age for end stage renal disease patients on heamodialysis was 44.1 and median age was 43 years. On the contrary, Najma, Minhas, Aslam, Abbas and Asad (2005) had an average age for ESRD patients on heamodialysis as 66 and the age ranged from 60 to 70 years. This difference could be a result of rising prevalence of diseases causing ESRD such as hypertension and diabetes among the younger generation in the developing countries.

Results of this study also showed that 5(5.9\%) had attained the primary seven years of education and below, 80(94\%) had attained at least two years in high school. This showed higher prevalence of ESRD among the educated patients. These results are similar to those of Nizam (2005) which showed that $53.3 \%$ had education above matriculation whereas $46.7 \%$ were below matriculation. However, higher level of education enables an individual to be literate, understand health education and health instructions. According to Golper (2002), low education level may lead to decreased adherence to heamodialysis and poor correlation with knowledge of disease

On income, 50(58.8\%) did not have any income. In most of developing countries, the patient provides bulk of the funds for heamodialysis therapy whereas in developed countries, the costs of heamodialysis are borne by the government (Alebiosu \& Ayodele, 2005).The current situation in Zimbabwe is such that ESRD patients are paying for heamodialysis. This situation makes heamodialysis treatment unavailable to all patients thus reducing heamodialysis adherence and adequacy to end stage renal disease patients.

Concerning duration of illness on heamodialysis, the results showed that m;mmk 19(22.4\%) had end stage renal disease for 3 months to 1 year, $19(22.4 \%)$ had end stage renal disease for 1 to 2 years, $18(21.2 \%)$ had end stage renal disease for 2 to 3 years, $1(1.2 \%)$ had end stage renal disease for 3 to 4 years, 10(11.8\%) had end stage renal disease for 4 to 5 years and $18(21.2 \%)$ had end stage renal disease for more than 5 years. Duration of disease or treatment is a determinant of adherence to heamodialysis in end stage renal disease patients. Cramer (1991) did a study and found out that adherence wanes with longer treatment. Treatment of end stage renal disease by heamodialysis is a lifelong saving procedure and adherence to heamodialysis might be affected by duration of end stage renal disease.

\subsection{Perceived Health Status}

The results of the perceived physical health questionnaire were attempting to establish perception of physical health among end stage renal disease patients attending heamodialysis at Parirenyatwa hospital a Central Hospital in Zimbabwe. Thirty-two respondents (37.6\%) perceive their health as poor and 41(48, 2\%) pereceived their health as fair. Only $12(14 \%)$ of the respondents perceived their physical health status as at least good. Only $11(13 \%)$ of the respondents perceived their health as improved in the past 3 months while the rest perceived their health as having remained static or had worsened. A total score of 67 points was the highest possible score expected in this study. According to the perceived physical health questionnaire, the mean score was 33.11.The results show that, the majority 53(62\%) of respondents had total perceived physical health scores below the mean and $32(38 \%)$ had total perceived physical health scores above the mean. According to the scale developed by the investigator, $82(96,5 \%)$ of respondents perceived their physical health as average. The perceived physical health scores were assessed according to categories of general perception of physical health, how perceived physical health affect activities done on a typical day, how physical health affect activities of daily living and to what extent had perceived physical health affected adherence to heamodialysis.

From the above results it is clear that ESRD patients had a fair perception of their physical health status. Activities of living are known to be greatly deranged and the patient is far from being independent in the performance of activities of living. These results support the findings by Kouidi (2004), who found out that ESRD patients have low functional capacity and physical limitations. According to Davies (2001), it is well documented that the physical health status of end stage renal disease is worse than that of the general healthy population. Noam et al (2005) also reinforced that patients with end stage renal disease perceive themselves as being unwell, physical health impaired due to heamodialysis and having reduced quality of life.

Sanjeek, Mittal \& Lori (2009), did a cohort study on self assessed physical function of heamodialysis patients. The study sought to evaluate the physical function in heamodialysis population as compared to the general population and other chronic illnesses. The results of the study showed that physical function in end stage renal disease patients is lower than in most other chronic illnesses. These findings also supported the study results.

In relation to how perceived physical health affect activities done on a typical day, the results showed that, $83(97.6 \%)$ are limited a lot by their physical health in performing vigorous activities such as lifting heavy objects and participating in strenuous sports. Morais (2004), suggested that because of signs and symptoms of end stage renal disease and heamodialysis, we need to understand that end stage renal disease patients suffer several physical limitations including limitations in performing vigorous activities, moderate activities, walking 
more than a kilometer and lifting of heavy objects and groceries. Therefore, the present study findings concur with the results from Morais (2004). Kouide (2004) concurs as his findings confirmed that ESRD patients have low functional capacity and physical limitations in their daily activities.

Kirsten (2001) conducted a study to estimate physical activity and functioning in ESRD patients. Results showed that ESRD patients on heamodialysis are less physically healthy than persons with normal kidney function. On personal cleansing and dressing, 26(30.6\%) reported having problems most of the time. The results showed that some end stage renal disease patients generally have problems in personal cleansing and dressing. Bezerra, Piantino \& Morais (2005) also found out that end stage renal disease patients usually presents with deficits in personal cleansing and dressing due to altered physical health. According to Mitch (2007), symptoms in end stage renal disease that affects personal cleansing and dressing includes, drowsiness, fatigue, general ill feeling, and muscle cramps. ESRD patients are therefore physically ill and they usually don't have the energies to bath and dress themselves, therefore need to be assisted.

Eating and drinking also posed challenges to most study participants in the present study. Fifty - nine (69.4\%) reported having difficulties most the time. According to Mitch, 2007, eating and drinking in end stage renal disease patients is affected by changes in dietary plans, restrictions in some type of food intake and restrictions in fluid intakes. Symptoms of ESRD that affects eating and drinking includes poor appetite, nausea, vomiting and bad breadth. On elimination, 71(83.5\%) reported having problems with associated with urination most of the times. Due to the pathologic process of ESRD patients continue to suffer elimination problems (Mitch, 2007). Elimination plays a major role in the everyday life. That is why ESRD patients have to be adherent to haemodialysis for the rest of their life to get rid of toxic substances in the body.

Concerning mobilization, $51(60 \%)$ reported having problems most of the time, $18(21.2 \%)$ reported having problems some of the time, $8(9.4 \%)$ reported having problems a little of the time and $8(9.4 \%)$ reported never having problems with this activity. According to the study results, $92 \%$ of end stage renal disease patients exhibit signs of altered mobility in carrying out their daily chores. Tedsco (2006) stated that most end stage renal disease patients are usually limited in body movements such as walking, jogging, exercising and carrying of groceries. This study reinforced these previous findings. According to Mitch (2007) symptoms of ESRD that affect mobility include weakening of the bones, joint disorders, and poor muscle tone and tissue swellings.

Fifty - one $(60 \%)$ reported having sleep problems most of the time. The prevalence of sleep disorders is high and this may contribute to an impaired quality of physical health (Mucen, Boisteau, Wirth \& Covic, 2007). It is also evident that sleep disorders are more frequent in heamodialysis populations than the general population (Sakkas, 2008). These two studies support the study findings which are showing high rate of sleep problems. Sleep disturbances are a possible consequence of the long process of ESRD and the exposure to the uremia milieu.

The high percentage of respondents with problems associated with working, 64(75.3\%) support earlier findings by Mitch (2007). According to Mitch (2007), ESRD patients exhibit symptoms that affect patients' ability to work and these include, muscle cramps, fatigue, pain, headaches, general body weakness and poor appetite among other things.

\subsection{Adherence to Haemodialysis}

Seventy-nine $(93 \%)$ respondents had missed at least one session with 52 respondents $(61 \%)$ missing most of the scheduled sessions and only $6(7 \%)$ attending all the haemodialysis sessions as scheduled. Fiftyseven $(67 \%)$ had rescheduled the prescribed haemodialysis sessions more than once. This confirms the findings by Tolkoff-Rubin (2008) that patient's non adherence to prescribed heamodialysis regimen is a pervasive problem in the end stage renal disease population.

Patient's apparent unwillingness to follow medication regimens or to adhere to medical treatment regimens more generally reflects a significant barrier to the effective management of most chronic disorders. As a result, prevalence of non adherence is estimated to be $50 \%$ or higher among patients following chronic medical regimens (Mitch, 2007). This has been shown in this study were more than 50\% of patients were not adherent to scheduled heamodialysis plan. Zimbabwe is a developing country and epidemiologic studies showed that adherence to heamodialysis is generally lower in developing countries than in the industrialized world (Remmuzi, 2000). The results confirmed lower adherence to heamodialysis in Zimbabwe. On the contrary, Bleyer, Hylander \& Sudo (1999) reported that missed dialysis treatments were virtually nonexistent in Japan, Sweden and only $2.3 \%$ of dialysis treatments were missed by patients in the United States of America. Although many factors may contribute to these differences, emphasis on patient independence in the USA might be a positive factor.

Adherence to heamodialysis remains a major problem in Zimbabwe and this also affects families and medical surgical nursing. Consequences of inadequate adherence pose more problems to the end stage renal disease patient, medical surgical nurses, families and society as a whole. 


\subsection{Perceived Physical Health and Adherence to Haemodialysis Among ESRD Patients}

Fifty-three (63\%) agreed to missed haemodialysis sessions because of feeling physically fit and 46 (54\%) agreed that it was not necessary to follow prescribed haemodialysis schedule. The results showed a weak significant weak negative correlation $(\mathrm{r}=-.44 \mathrm{p}<.01)$ between perceived physical health and adherence to heamodialysis among end stage renal disease patients. As perceived physical health increases in the end stage renal disease patients, adherence to heamodialysis decreases.

Regression analysis showed $\mathrm{R}^{2}$ of .197 which shows the impact or effect of the independent variable (perceived physical health) on the dependent variable (adherence to haemodialysis). The effect of the independent variable accounts for $19.7 \%$ of the variance in adherence to haemodialysis. Therefore, $19.7 \%$ of the changes in adherence to haemodialysis are due to perceived physical health. The significant negative effect $(b=-$ $.104, \mathrm{p}<.01)$ represents a change in adherence for every unit change in perceived physical health. The significant standardized coefficient $\mathrm{b}(-.44 \mathrm{p}<.01)$ indicates the relevant importance of perceived physical health as an independent variable. The bigger the value, the more important the independent variable is in terms of its contribution towards the dependent variable. Perceived physical health is therefore less important in terms of its contribution towards adherence to haemodialysis in this study sample.

The findings of this study are reinforced by Mapes, Gresham \& Bommer (2004). These investigators showed that perceived physical wellbeing of end stage renal disease patients have been seen to affect their adherence to heamodialysis. Elsevier (2009) concurred and further stated that patient's beliefs about their physical health are important because they influence adherence and adjustment to heamodialysis. A study by Obialo, Hunt, Bashir and Zanger (2012)which revealed that non-adherence was more prevalent in younger patients who may feel they are physically healthier than the older patients thus can get away with missed haemodialysis sessions, also supporst results of this study. However, the present study results contrast with those by Koudi (2004) who found out that ESRD patients display physical health disturbances as well as non adherence to heamodialysis treatment.

\subsection{Nursing Practice}

\section{Nursing Implications}

It appeared that lower adherence to heamodialysis was partially due to false sense of good physical health among end stage renal disease patients. If this situation is not addressed the positive effects of haemodialysis will not be realized. More and more patients will continue to deteriorate and even require more aggressive therapies. The more aggressive therapies may entail more dialysis hours per week or use of stronger dialysate solutions. Both these strategies call for more resources as well as increasing workload on the nurses.

\subsection{Nursing Education}

The study equips practising nurses with adequate knowledge on subtle areas pertaining to ESRD patients' perceptions and behaviours. Medical surgical nurses need to be given adequate evidence based knowledge to enable them to appropriately address ESRD patients' issues.

\subsection{Nursing Research}

A significant but weak negative correlation was found between perceived physical health and adherence to heamodialysis. Perceived physical health only explained $19.7 \%$ of the variance in adherence to haemodialysis. There should be more contributory factors leading to varying adherence levels among ESRD patients. Important implications to nursing research are inevitable as nursing research is called for to set up more inquiry into the major contributory factors to dialysis adherence problems among ESRD patients.

\subsection{Nursing Administration}

Nurse Administrators are the backbone for providing facilities to improve knowledge on the importance of adhering to the prescribed haemodialysis. Administrative policies should be formulated at the large scale to address issues that affect adherence to haemodialysis.

\section{Conclusion}

The level of adherence to heamodialysis was low for 83 (97.6\%) of participants. In addition, 82 (96.5\%) of participants perceived their physical health to be average. Pearson correlation analysis showed a weak negative correlation $(\mathrm{r}=.44, \mathrm{p}<.01)$ of perceived physical health and adherence to heamodialysis among ESRD patients. The results imply that to a small extent, as the level of perceived physical health increases, adherence to heamodialysis decreases. The weak relationship between the study variables was further supported by a linear regression analysis which showed perceived physical health can only explain $19.7 \%$ of the variance on adherence to haemodialysis. 


\section{References}

[1]. Alebiosu, C.O., \& Ayodele, O.E. (2005) the global burden of chronic Kidney disease and the way forward. Ethnic Disease, 15: 418 $-423$

[2]. Christensen, A. J. (2000). Patient by treatment context interaction chronic diseases: A conceptual framework for the study of patient adherence: Psychosomatic medicine. St Louis: Mosby.

[3]. Cukor, D. (2007). Health related quality of life in end stage renal disease population. Journal of nephrology, 6( 5); 1213-16.

[4]. Elsevier, B.V. (2009). Heamodialysis patients' beliefs about renal failure and its treatment, patient counseling and education. American Journal of Medicine. 53 (2):189 -196.

[5]. Golper, T. (2002). Patient education; can it maximize the success of therapy. Nephrology Dial Transplant, 16 supplement 7: 20 24.

[6]. Hays, R.D., Kallich, J.D., Mapes, D.L., Coons, S.J., and Carter, W.B. (2004) Development of the Kidney Disease Quality of Life (KDGOL) . Journal of quality of life research (10): $329-338$.

[7]. Kouidi, E. (2004). Health related quality of life in end stage renal disease patients; the effects of renal rehabilitation. Clinical Nephrology. Supplement, 14: 7792

[8]. Kurtner, N.G. (1994). Assessing ESRD patients functioning and well being; measurement approaches and implications for clinical practice. American journal of kidney disease, 16 (4): 16-19.

[9]. Mitch, W.E. (2007). Chronic kidney disease in: Goldman, L., Ausiello, And D., eds. Goldman: Cecil Median. (23 ${ }^{\text {rd }}$ ed.). Philadelphia: Saunders.

[10]. Najma, N., Minhas, A.F., Aslam, F., Abbas, S., \& Assad, N. (2005). Relationship between psychological wellbeing, depression anxiety and quality of life in ESRD patients having dialysis. Journal of nephrology,5 (6) 34 -38.

[11]. Obialo, C.I., Hunt, W.C,Bashir, K and Zager, P. G (2012) Relationship of missed and shortened treatments to hospitalization and mortality: Observation from a US dialysis network, Clinical Kidney Journal (5): 315-319

[12]. Remuzzi, G. (2001) A research program for COMGAN. ISN News.

[13]. Barsoum, R,S (2013) Burden Of CKD; North Africa. Kidney international Supplements. Volume 3 (2): 164-166. Doi; $: 10.1038 /$ Kisup.2013.5

[14]. Naicker, S (2013) End Stage Renal Disease in sub-Saharan Africa. Official journal of the international society of nephrology. Kidney international supplements 3, 161-163

[15]. Vivekanand JHA, Guillermo G, Kunitoshi, I, Zou, 1, Saraladev, N (2013) Chronic kidney disease; Global dimension and perspectives. The lancet Publication. Volume 382 issue 9888 page 260-272. Doi :10.1016/S0140-6736 (13) 60687-X

[16]. Farag, Y. M.K, Kari, J.A, Singh, A.K (2012) Chronic kidney disease in Arab world : A call for action. Nephron clinical practice. Issue 121 page C120-C123. Doi : 10-1159/000345149

[17]. Lozano, R, Naghavi, K, Foreman, K (2010) Global and regional mortality from 235 causes of death for 20 age groups in 1990 and 2010; Asystemic analysis of global burden of disease study 2010. The Lancet, volume 380. Issue 9859 pages 2095-2128 